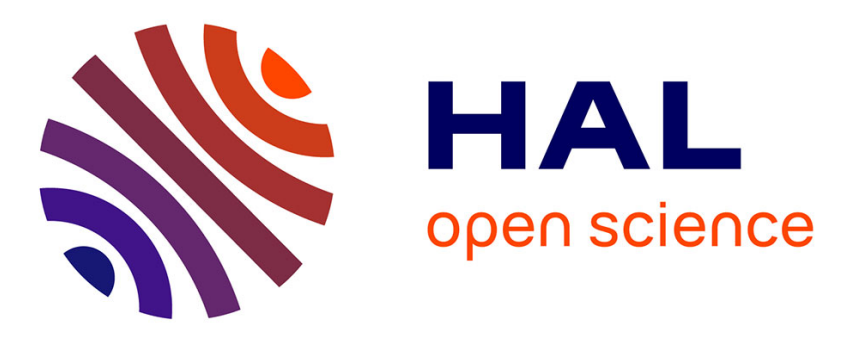

\title{
Assessment of Ge-doped optical fibre as a TL-mode detector
}

\author{
Mourad Benabdesselam, Franck Mady, Sylvain Girard
}

\section{To cite this version:}

Mourad Benabdesselam, Franck Mady, Sylvain Girard. Assessment of Ge-doped optical fibre as a TL-mode detector. Journal of Non-Crystalline Solids, 2013, 360, pp.9. hal-00780090

\section{HAL Id: hal-00780090 https://hal.science/hal-00780090}

Submitted on 23 Jan 2013

HAL is a multi-disciplinary open access archive for the deposit and dissemination of scientific research documents, whether they are published or not. The documents may come from teaching and research institutions in France or abroad, or from public or private research centers.
L'archive ouverte pluridisciplinaire HAL, est destinée au dépôt et à la diffusion de documents scientifiques de niveau recherche, publiés ou non, émanant des établissements d'enseignement et de recherche français ou étrangers, des laboratoires publics ou privés. 


\title{
Assessment of Ge-doped optical fibre as a TL-mode detector
}

\author{
M. Benabdesselam ${ }^{a}{ }^{*}$, F. Mady ${ }^{a}$, S. Girard ${ }^{b}$ \\ ${ }^{a}$ Université de Nice-Sophia Antipolis, LPMC UMR CNRS 7336 \\ Parc Valrose, 06108 Nice Cedex 2, France \\ ${ }^{\mathrm{b}}$ CEA, DAM, DIF \\ Bruyères-le-châtel, 91297, Arpajon Cedex
}

\begin{abstract}
This study analyses the thermally stimulated luminescence or thermoluminescence (TL) glow curve between 300 and $773 \mathrm{~K}$ of germanium-doped silica optical fibre. A main glow peak at $530 \mathrm{~K}$ with a characteristic spectral emission centred at $400 \mathrm{~nm}$ is found. Both features are particularly suitable for dosimetry.

Thus, an investigation by the TL technique of some first clinically relevant features of a TL sensor like the doseand dose rate-responses is examined. The presented studies show that germanium doped silica fibres have potential dosimetric properties and should be excellent TL-mode detectors in instances of radiotherapy (clinical dosimetry) and in-vivo radiation dosimetry as well in the field of nuclear facilities.
\end{abstract}

PACS Keywords: dosimetry, radiation, silica, thermoluminescence, optical fibres

* Corresponding author. Tel: 0033492076332 fax: 0033492076756

Email address: ben@unice.fr (M. Benabdesselam) 


\section{Introduction}

In view of their high TL sensitivity, storage stability and reliability, the outstanding advantages presented by Gedoped optical fibres are interesting for application in ionising radiation dosimetry and have to be explored.

With the achievement of the modified chemical vapour deposition (MCVD) process, reproducible and large-scale production, high quality optical fibres are now available at moderate cost [1]. In addition, optical fibres are chemically inert, physically robust and biocompatible and their re-use and sterilisation are obtained by simple heating.

For many years and for several reasons, many authors have done a large body of work on the radiation effects on Ge-doped silica and on silica based fibres [2,5]

During the last decade, a number of research groups have investigated the TL response of commercially available silica based optical fibres for application in the field of photon and electron radiation dosimetry [6,7] but up to the absorbed dose of $10 \mathrm{krad}$ and the comparison against one classical crystalline TLD material is made just in terms of TL sensitivity. At present, clinical applications based on TL properties of Ge-doped optical fibres appeared [8]. Classical thermoluminescence dosimeters (TLD) use the property of TL signal relative, for practical reasons; to deep trap levels stable at room temperature (RT), which the population increases with the radiation-absorbed dose. In this technique, the deep level acts as "a memory cell" of the ionising radiation dose. The trapping properties of the deep level in the TL process and its concentration are among the critical parameters that control the behaviour of the dosimeter.

\section{Experimental setup}

The GeD2 fibre studied here is produced by iXFiber SAS from a preform made by MCVD process. The fibre core diameter is $62.5 \mu \mathrm{m}$ with a two-steps Ge-doping. The first step is doped with $4,5 \mathrm{wt} . \%$ and the second is at a level of about 9 wt.\%. Typical levels of chlorine impurity and $\mathrm{OH}$-groups in the fibre are $\sim 1200 \mathrm{ppm}$ and $\sim 60 \mathrm{ppb}$ respectively $[9,10]$.

Because of heating, the TL analysis requires the polymer coating removal on a fibre length. The obtained silica material is cleaned, cut in stalks of some millimetres in length and then put on a $10 \mathrm{~mm}$ diameter aluminium cupel. Irradiations were achieved at RT by means of an X-ray tube (Cu target, $45 \mathrm{kV})$ at different dose rates. One minute after the end of irradiation, TL readout was carried out from RT up to $750 \mathrm{~K}$ with a linear heating rate of $1 \mathrm{~K} / \mathrm{s}$ and the signal was recorded by means of a $\mathrm{S} 13$ response photomultiplier tube $(250-600 \mathrm{~nm})$. 
An optical multichannel analyser (OMA) was used to detect the spectral distribution of the TL peak. The analyser consists of an optical fibre (fused silica) and a Chromex 250 IS spectrograph equipped with a CCD matrix (Princeton Instruments), the spectral response of the system is within the range $200-1100 \mathrm{~nm}$.

\section{Results}

\section{1. Trapping and luminescence parameters}

Among various studied optical fibres, Ge-doped one and named GeD2 is by far, the sample providing the most sensitive TL yield by comparison to the undoped fibre or to those containing impurities like Al, F, P, or RE. The typical glow curve of this fibre is composed by one shoulder at around $360 \mathrm{~K}$ and a main broad component $(\Delta \mathrm{T}=$ $138 \mathrm{~K}$ ) peaking at $530 \mathrm{~K}$ (Fig. 1). The broadening of TL glow peaks result from the spread of trap levels reflecting the amorphous structure of the sample unlike a crystal where point defects give rise to single localised levels and to the corresponding narrow TL peaks. The shoulder at $360 \mathrm{~K}$, always observed by TL in all tested silica based fibres, is probably due to a common intrinsic defect in silica, acting as a shallow trap level within the band gap of the material. It has also been observed in quartz and fused silica, as ubiquitous peak and assigned to an intrinsic defect complex implying $\mathrm{Al}$ and other impurities [11]. One focuses our study on the main TL peak at $530 \mathrm{~K}$, which is particularly well suited for TL dosimetry. Henceforth, this peak will be referred to as the "dosimetric peak".

Fig. 2 represents the emission spectrum of the dosimetric peak where the inset corresponds to the colour-map of the wavelength resolved TL measurement on a GeD2 fibre. Throughout the temperature range one observe only one emission, which consists of a blue-violet broadband luminescence centred at around $3.1 \mathrm{eV}(400 \mathrm{~nm})$ with a full width at half maximum $(F W H M=0.44 \mathrm{eV})$. This well known emission has been ascribed to the luminescence of the two-fold Ge centre (=Ge:) [12] and identified as a trapped-electron centre [13].

\section{2. Dosimetric characteristics}

\section{2. 1. TL sensitivity}

Fig. 3 shows the TL responses of the GeD2 fibre and of two crystalline TLD phosphors, LiF: Mg, Ti (TLD700) and $\mathrm{Al}_{2} \mathrm{O}_{3}$ : C (TLD500). These latter are the commercially available TL dosimeters (TLD), commonly used as in vivo radiation dosimeters for patients undergoing radiotherapy. The dosimetric peak of TLD 500 and TLD 700 is located at 435 and $490 \mathrm{~K}$ respectively.

\section{2. 2. Dose and dose rate TL response}


$T L$ response of GeD2 as a function of the absorbed dose is shown in Fig. 4. For a dose rate of $60 \mathrm{rad} / \mathrm{s}$, it presents a good linearity up to $60 \mathrm{krad}(600 \mathrm{~Gy})$. The linearity measurements were also continued for a higher dose rate of $490 \mathrm{rad} / \mathrm{s}$ (case b) to higher doses and showed remarkably good behavior up to $90 \mathrm{krad}$. The absorbed doses can be, within the experimental error, assessed independent of the dose rate, particularly in the range of dose up to 1 krad (10 Gy) where it is essential for the fibre to be used as a clinical dosimeter without using any calibration.

\section{2. 3. Repeatability and reproducibility}

Repeatability corresponds to the deviation of TL response for an individual fibre while the reproducibility is the TL response dispersion between fibres. The repeatability of the fibre under test for a given dose was calculated after five successive readout cycles and it had a percentage standard deviation at 2,8 \%. Reproducibility tests made on only two fibres are very satisfactory but batch variation due to the fibre manufacturing process was not performed.

\section{2. 4. TL quenching, time-dependent and optical fading}

Among the important features required for dosimetry by $T L$, is the ability of the dosimeter to entirely memorize the absorbed dose over a long period of time. The loss of the dose information, even partly, is called fading and may be induced at RT either by thermally excited electronic transitions (time-dependent fading) or optically induced by laboratory lighting (optical fading). With respect to the TL intensity obtained just after irradiation, the calculated percentage deviations are of $5 \%, 8$ hours post irradiation for thermal fading and $5 \%, 1$ hour post irradiation for the optical one.

Another feature concerns the effect of the heating rate variation on the TL response of the dosimetric peak. The use of different heating rates leads to a shift of the temperature maximum of the peak. This effect is well known in TL and was exploited by many authors to extract the trapping parameters of the TL peak [14].

In principle, the area under the TL peak is independent of the heating rate. By contrast, this area sometimes decreases with increasing heating rate if thermal quenching occurs. This could be inconvenient for comparative works between dosimetrists. This phenomenon, known as thermal quenching [15] is almost completely absent in the glow curve of GeD2 (Fig. 5).

\section{Discussion}

GeD2 fibre shows high TL response sensitivity of the main peak, which is ideally located in temperature (Fig. 1). Indeed, the temperature of $530 \mathrm{~K}$ is high enough to not empty traps linked to this peak (during storage at RT) and at the same time, sufficiently low as not to be affected by the black-body radiation appearing when heating 
increases. The trap parameters relative to this peak have been determined: the mean activation energy and frequency escape factor are respectively $E \approx 1,46 \mathrm{eV}$ and $\mathrm{s}=1,3 \times 10^{12} \mathrm{~s}^{-1}$.

The spectral distribution of this dosimetric peak shown in Fig. 2, is centred at $400 \mathrm{~nm}$, ie in the middle of the spectral band width of all UV-Vis PM tubes used in TL dosimetry. Moreover, the emission bands of the TL peaks of commercial TLD 500 and TLD 700 dosimeters are respectively located at 420 and $400 \mathrm{~nm}$, like GeD2 fibre.

TL sensitivity, peak position and spectral response constitute the first main required properties of the TL-mode detector. The GeD2 fibre seems to fit these properties and might be a potential candidate for some basic dosimetry tests.

The TL response as a function of the absorbed X-ray dose is presented on Fig. 3 for two dose rates (a: 60 and b: $490 \mathrm{rad} / \mathrm{s})$. We note that for both rates, the TL response shows a quite linear behaviour $\left(R^{2}=0,998\right)$ within a wide dose range and no obvious effect of the dose rate on the TL response of GeD2 is observed even if the rates variation occurs within only one decade (that's what makes our X-ray tube). Anyway, it is useful to note that at low dose levels (below1 krad) where clinical application is concerned, the two curves are superimposed.

The repeatability value of $2.8 \%$ is within the experimental uncertainty of the TL response sensitivity but might be considered as relatively high for an application in medical environment since the IAEA protocol 398 recommends a deviation of $0.5 \%$ [16]. Nevertheless, TLD700 is marketed with repeatability specification within $2 \%$ but subject to a thermal treatment of $1 \mathrm{~h}$ at $400^{\circ} \mathrm{C}$ and $2 \mathrm{~h}$ at $100^{\circ} \mathrm{C}$ before each use while the fibre is reusable instantly and does not require such a protocol.

Concerning the reproducibility, given the length of fibre at our disposal, measurements were done only on two samples both from the same batch. Reproducibility will be correctly estimated when the batch variation due to the fibre manufacturing and drawing processes will have been studied.

The TL response of irradiated GeD2 fibre and stored in the dark faded by $5 \%$ in the first $8 \mathrm{~h}$. This time-dependent fading is of the same order of magnitude than that of TLD500 and TLD700.

Under fluorescent light, the GeD2 fibre is slightly sensitive (5\% of loss, $1 \mathrm{~h}$ post irradiation) while TLD700 is not but GeD2 fibre outperforms the TLD500, for which the signal of the stored dose information fell by $83 \%$ in only 10 min of exposure [17].

Finally, curves in Fig. 5 clearly show that the GeD2 fibre is not subject to thermal quenching since the TL response still remains constant in the heating rate range $1-10 \mathrm{~K} / \mathrm{s}$ unlike TLD 500 where it manifests by a decay of $40 \%$ just in the range $0.5-2 \mathrm{~K} / \mathrm{s}[18]$. 


\section{Conclusion}

The results presented here clearly show that GeD2 optical fibre appears to meet all the various dosimetric criteria required by a TL-mode detector. The combination of a high sensitivity, a good linearity of response over a wide range of doses (up to $90 \mathrm{krad}$ ), an independence of the dose rate, make GeD2 optical fibre, a potential cheap, easy-to-use TL-mode detector.

Furthermore, even if TLD700 is ease of handling, it requires for its correct operation, an annealing protocol that can be cumbersome and time consuming for medical routine use while GeD2 fibre can be re-used without any particular regeneration treatment.

Irradiated TLD500 should never be exposed to fluorescent light. The light-induced fading is so significant that it requires a proper handling and storage while the sensitivity to light of GeD2 is low. Another interesting feature of GeD2 fibre is the absence of thermal quenching effect on its response while it strongly affects the TL sensitivity of TLD500.

The control of the deep level linked to the dosimetric peak within the silica band gap, by optimising the Ge impurities content in addition to a larger core fibre, might lead to a new kind of much cheaper TL-mode detectors with by all the odds, some dosimetric characteristics even better than the two compared commercial TLDs.

The effects of synthesis parameters as well as the drawing conditions on the TL peak response to $X$ rays and other types of radiations as well, are in progress and seem to confirm the suitability of GeD2 optical fibre in the TLmode detection. 


\section{References}

[1] K.T.V. Grattan, B.T. Meggit, Optical Fiber Sensor Technology. Advanced applications, Bragg gratings and Distributed sensors. Kluwer Academic Publishers. The Netherlands, (2000), p. 2

[2] A. Alessi, S. Girard, M. Cannas, S. Agnello, A. Boukenter and Y. Ouerdane, Optics Express, 19 (2011), p. 11680

[3] A. Alessi, S. Agnello, S. Grandi, A. Parlato, F.M. Gelardi, Phys. Rev. B 80 (2009), p. 014103

[4] M. Fujimaki, T. Katoh, T. Kasahara and Y. Ohki, J. Phys. Condens. Matter 11 (1999), p. 2589

[5] E.J. Friebele, D.L. Griscom, and G.H. Sigel, J. Appl. Phys. 45 (1974), p. 3424

[6] A.A. Youssef, Y.M. Yamin, D.A. Bradley, Radiat. Phys. Chem., 61 (2001), P. 409

[7] S. Hashim, S. Al-Ahbabi, D.A. Bradley, M. Webb, C. Jeynes, A.T. Ramli, H. Wagiran, Applied Radiation and Isotopes, 67 (2009), p. 423

[8] A.T. Abdul Rahman, R.P. Hugtenberg, Siti Fairus Abdul Sani, A.I.M. Alalawi, Fatma Issa, R. Thomas, M.A. Barry, A. Nisbet, D.A. Bradley, Applied Radiation and Isotopes, 70 (2012), p. 1436

[9] A. Alessi, S. Girard, C. Marcandella, S. Agnello, M. Cannas, A. Boukenter, Y. Ouerdane, Journal of NonCrystalline Solids 357 (2011), p. 1966

[10] S. Girard, Y. Ouerdane, G. Origlio, C. Marcandella, A. Boukenter, N. Richard, J. Baggio, P. Paillet, M. Cannas, J. Bisutti, J.P. Meunier, R. Boscaino, IEEE Transactions on Nuclear Science, 55 (2008), p. 3473

[11] S.W.S. McKeever, Thermoluminescence of Solids, Cambridge University Press (2005)

[12] L. Skuja, "Optical Properties of Silica" in Defects in $\mathrm{SiO}_{2}$ and Related Dielectrics: Science and Technology, p108 Edited by G. Pacchioni, L. Skuja and D. L. Griscom, 2000

[13] D.L. Griscom, J. NonCryst. Solids, 357 (2011), p. 1945

[14] R. Chen, L. Winner, J. Appl. Phys., 41 (1970), p. 5227

[15] D. Curie, Luminescence in Crystals, Methuen, London (1963)

[16] IAEA Tech. Rep. Ser, 398 (2000), Vienna 100 (2002)

[17] M. Moscovitch, R. A. Tawil, M. Svinkin, Radiat. Prot. Dosim, 47 (1993), p. 251

[18] S. Vinceller, G. Molnar, A. Berkane Krachai, P. lacconi, Radiat. Prot. Dosim., 100 (2002), p. 79 
Figures captions

Fig. 1. Typical TL glow curve of GeD2 fibre recorded at $1 \mathrm{~K} / \mathrm{s}$ after X-ray irradiation at RT.

Fig. 2. Emission spectrum of the dosimetric peak observed on GeD2 optical fibre after X-ray irradiation at RT. The inset shows the colour-map of the wavelength resolved TL measurement throughout the temperature range.

Fig. 3. TL superposition of the GeD2 optical fibre, TLD500 and TLD700 under the same conditions of irradiation and readout.

Fig. 4. TL response of the dosimetric peak as a function of the X-ray dose at 60 (a) and $490 \mathrm{rad} / \mathrm{s}$ (b)

Fig. 5: TL curves obtained on GeD2 fibre for heating rates of 1 and $10 \mathrm{~K} / \mathrm{s}$ after the same $\mathrm{X}$ irradiation.

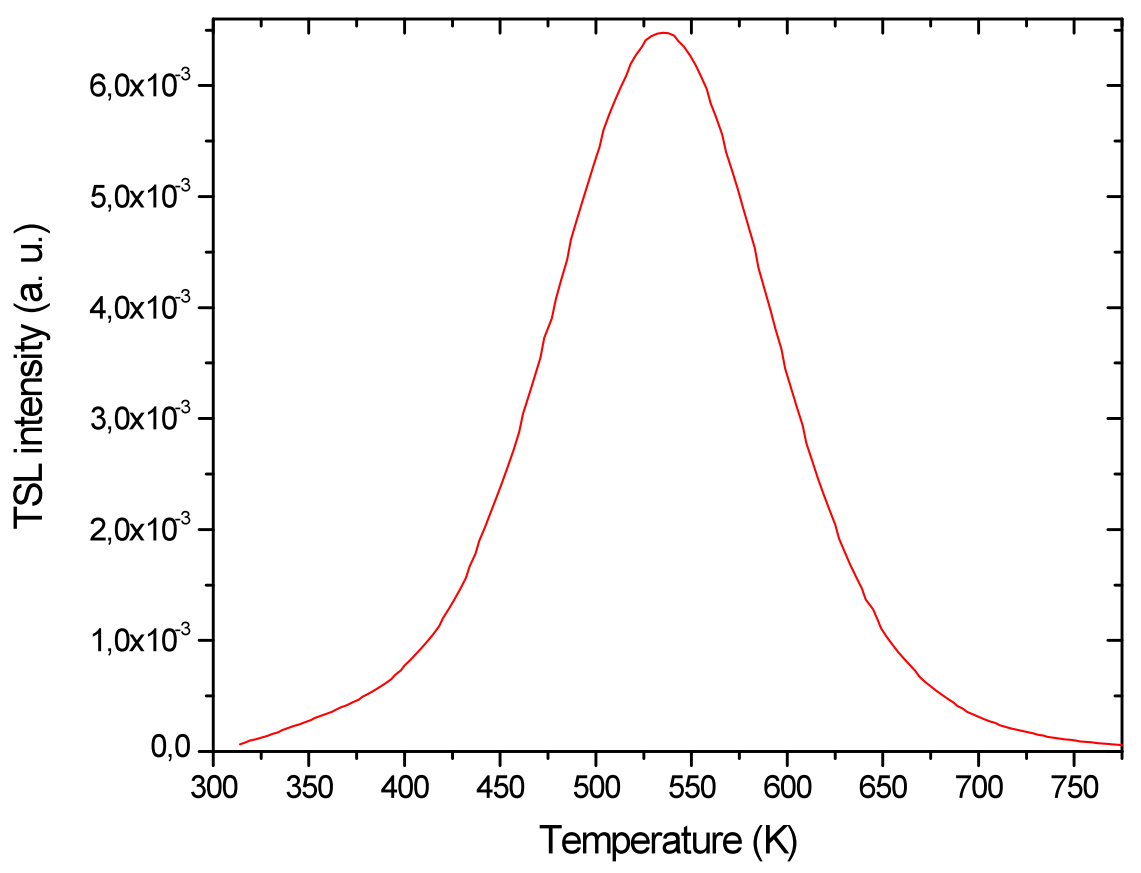

Fig. 1 


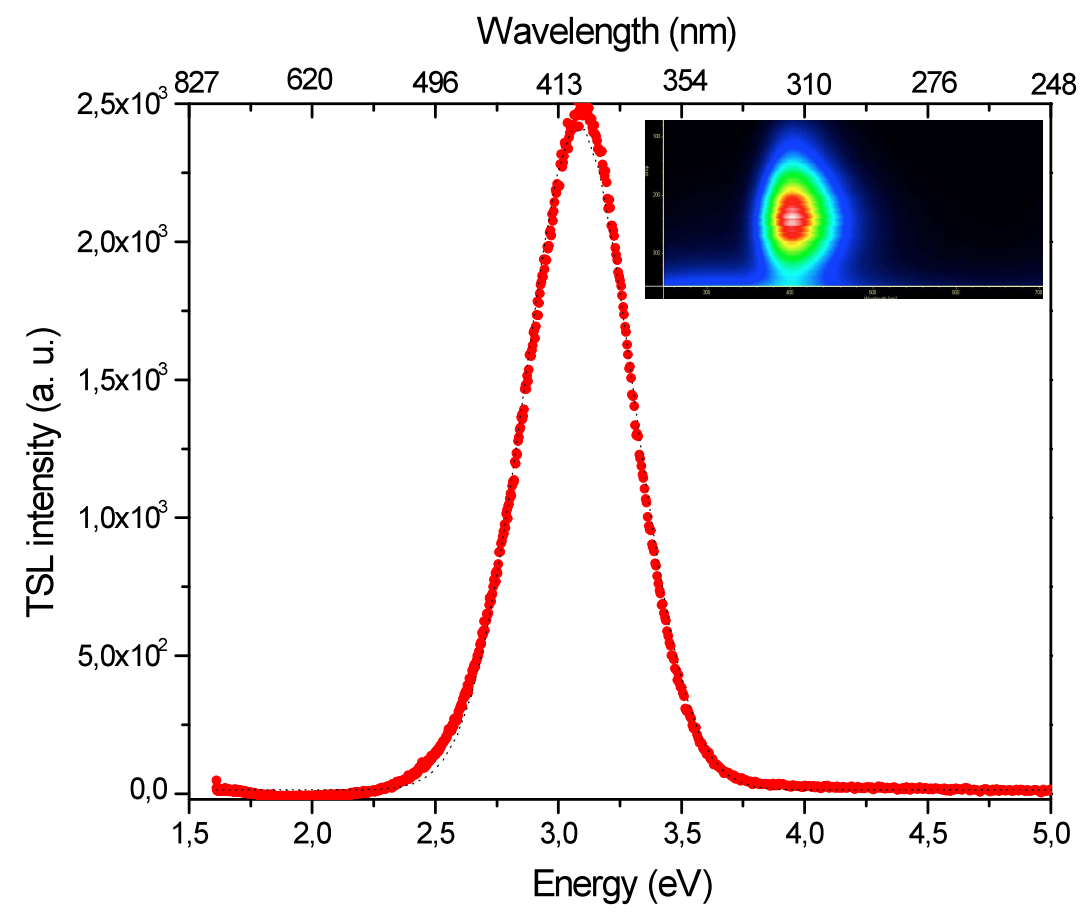

Fig. 2 


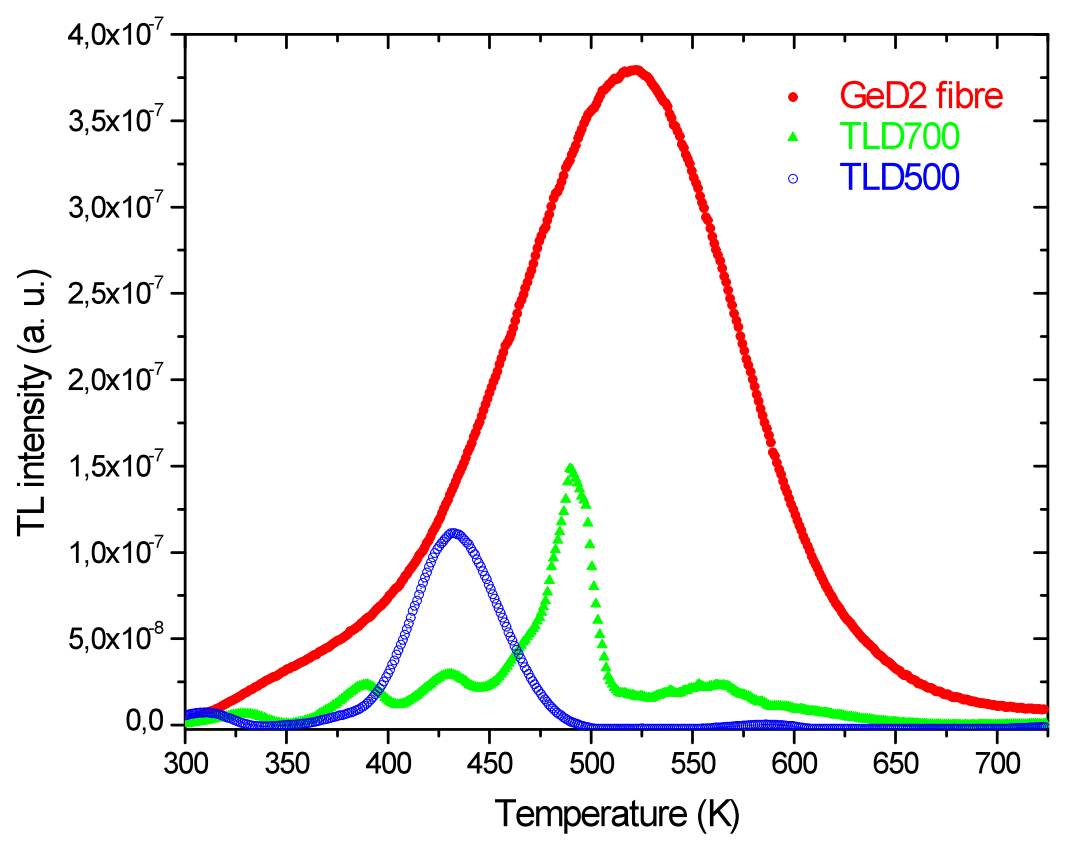

Fig. 3

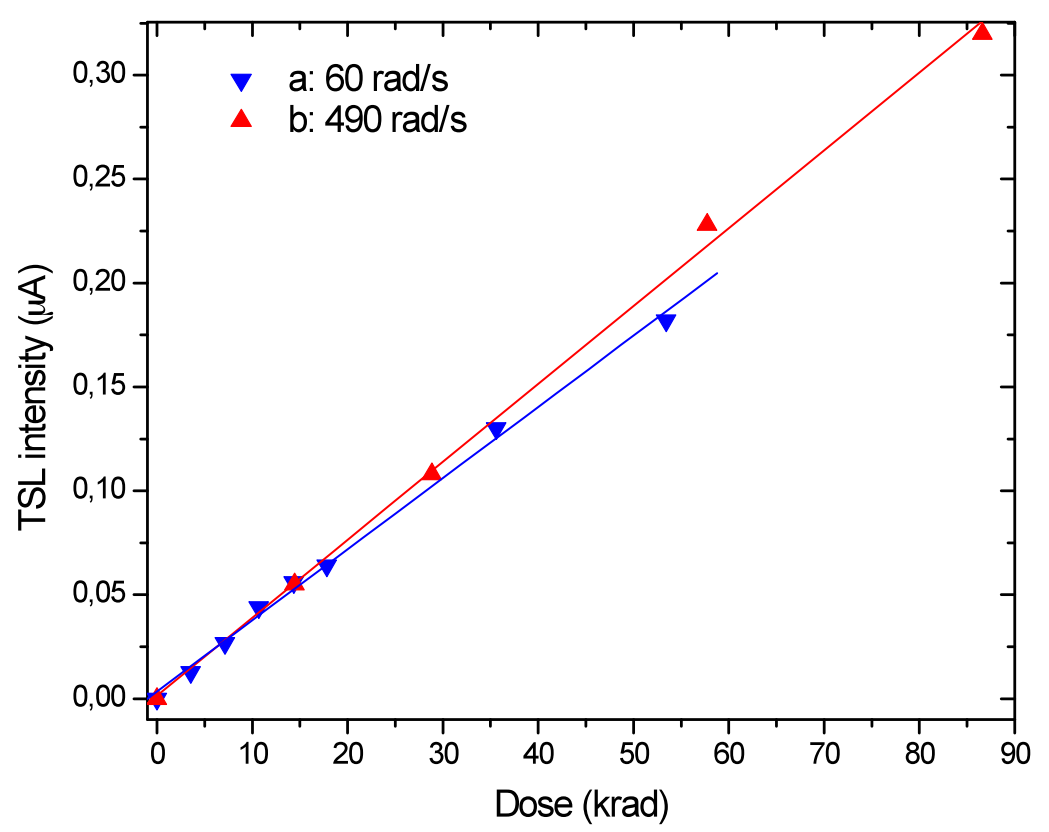

Fig. 4 


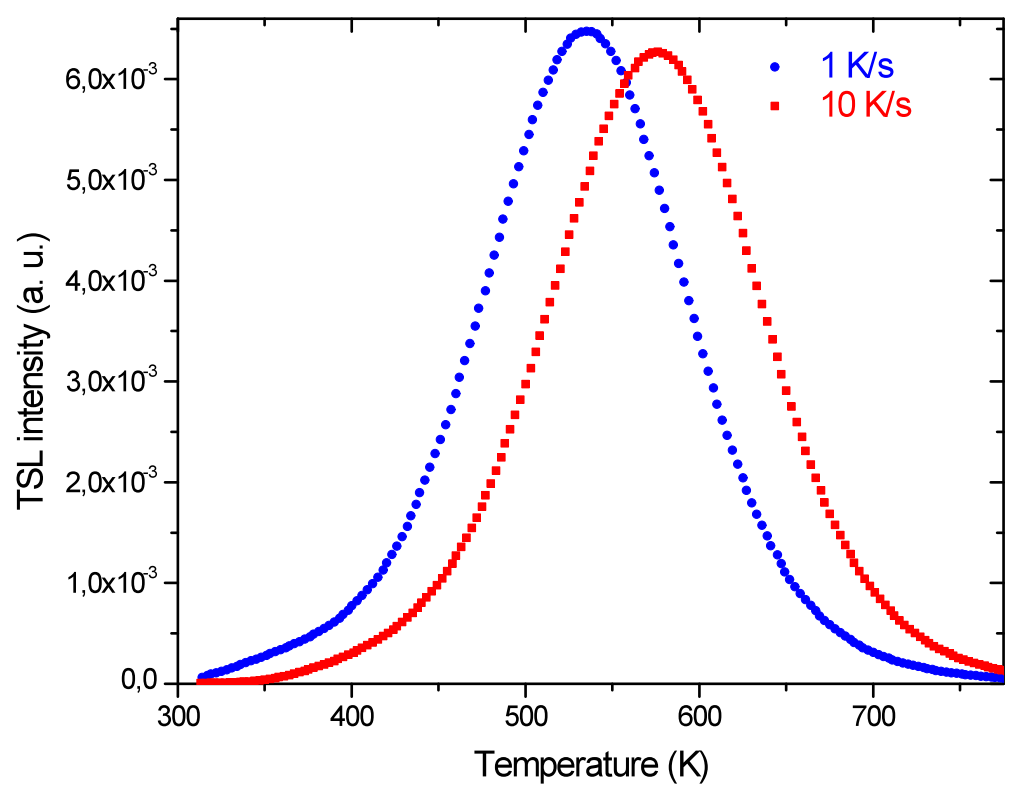

Fig. 5 